\title{
Radial basis integral equation method for Navier-Stokes equations
}

\author{
T. T. Bui \& V. Popov \\ Wessex Institute of Technology, Environmental and Fluid Mechanics, \\ Southampton, UK
}

\begin{abstract}
A meshless BEM approach has been developed, which has been applied for solution of the Navier-Stokes equations. The approach subdivides the original problem domain in a number of circular sub-domains around nodes used to represent the solution of the problem. Dual reciprocity method has been used to convert the domain integrals into integrals over the boundary of the sub-domains. Six equations are solved in 2D to obtain the solution of the Navier-Stokes equations, of which two are for solving the velocity components, one for pressure and the remaining equations are for solving for stresses. The developed formulation has been tested on the lid-driven cavity problem and the results have been compared to the results of Ghia et al., showing good agreement.

Keywords: Meshless method, integral equations, circular sub-domains, radial basis functions, Navier-Stokes equations.
\end{abstract}

\section{Introduction}

Meshless approaches based on the integral equations are receiving increased attention due to their accuracy associated with the integral equations methods, of which the most widely used so far has been the BEM, and the flexibility they offer as the meshing requirements are either eliminated or largely reduced.

The Local Boundary Integral Equation (LBIE) method [2,3] uses domain decomposition into a large number of circular sub-domains, with the source point in the centre of the circle. The LBIE uses the concept of "companion solution" in order to eliminate the single layer integral from the local boundary integral equation, leaving the potential field as the only unknown in the equations. For source points that are located on the boundary of the given problem part of the local circular bound- 
ary is replaced by the part of the global boundary and the integrals are evaluated over this part of the global boundary and remaining part of the circle.

Recently [4] LBIE has been employed for solution of the Navier-Stokes equations by using the velocity-vorticity formulation in combination with the radial basis functions (RBFs) used for interpolation of the field variables over the circular boundaries of the sub-domains.

Though the present formulation may seem similar to the LBIE in certain aspects, overall it is a fundamentally different approach. Similarly to the LBIE it is implemented over circular sub-domains where the source points are placed in the centres of the circles and uses RBFs for interpolation of the field variables over the circular boundary. However, the present approach is implemented using the velocitypressure formulation, it does not use the companion solution concept and does not need any integration over the boundary. More on the current formulation can be found in Bui and Popov [5].

\section{Velocity-pressure formulation}

For the incompressible flow, the equation of continuity is

$$
\nabla \cdot \boldsymbol{u}=0
$$

The conservation of momentum for an incompressible fluid is expressed as:

$$
\rho \frac{\partial u_{i}}{\partial t}+\rho u_{j} \frac{\partial u_{i}}{\partial x_{j}}=\frac{\partial \sigma_{i j}}{\partial x_{j}}+\rho F_{i}
$$

where $u_{i}$ is the component of the velocity vector along the $i$ direction, $\rho$ is the density and $F_{i}$ is the net body force along the $i$ direction; $\sigma_{i j}$ is stress tensor corresponding to the flow $\boldsymbol{u}, p$. For Newtonian fluid

$$
\sigma_{i j}=-p \delta_{i j}+\mu\left(\frac{\partial u_{i}}{\partial x_{j}}+\frac{\partial u_{j}}{\partial x_{i}}\right)
$$

where $p$ is the fluid pressure, $\delta_{i j}$ is the Kronecker delta and $\mu$ is the viscosity coefficient.

The integral representation for Navier-Stokes equations for a given point $x$ inside the domain $\Omega$ bounded by boundary $S$ is given by Ladyzhenskaya (1963) [6] as:

$$
u_{i}(x)=\int_{S} t_{k i}^{*}(x, y) u_{i}(y) d S_{y}-\int_{S} u_{k i}^{*}(x, y) t_{i}(y) d S_{y}+\int_{\Omega} u_{k i}^{*}(x, y) g_{i}(y) d \Omega
$$

where $g_{i}=\rho u_{j} u_{i, j}$ are convective terms; $t_{i}=\sigma_{i j} n_{j}$ are the traction components, $n_{j}$ is the outward normal vector; $u_{i}^{k}$ is the velocity field fundamental solution of 
the Stokes equations. In two dimensions, $u_{k i}^{*}$ is given as [6]

$$
u_{k i}^{*}(x, y)=-\frac{1}{4 \pi \mu}\left[\ln \left(\frac{1}{r}\right) \delta_{i k}+\frac{\left(x_{i}-y_{i}\right)\left(x_{k}-y_{k}\right)}{r^{2}}\right]
$$

with a corresponding pressure $q^{k}$ which is given by

$$
q^{k}(x, y)=-\frac{1}{2 \pi} \frac{\left(x_{k}-y_{k}\right)}{r^{2}}
$$

where $r=|x-y|$. The fundamental traction $t_{k i}^{*}(x, y)$ is defined as

$$
\begin{aligned}
& t_{k i}^{*}(x, y)=\sigma_{i j}^{\prime}\left(\boldsymbol{u}^{k}(x, y), q^{k}(x, y)\right) n_{j}(y) \\
& t_{k i}^{*}(x, y)=-\frac{1}{\pi r} \frac{\left(x_{i}-y_{i}\right)\left(x_{k}-y_{k}\right)\left(x_{j}-y_{j}\right)}{r^{3}} n_{j}
\end{aligned}
$$

The DRM approximation was introduced to express the domain integral in (4) in terms of equivalent boundary integrals. The convective term is expanded in the form

$$
g_{i}(x)=\sum_{m=1}^{N+A} f^{m}(x) \alpha_{l}^{m} \delta_{i l} .
$$

The coefficient $\alpha_{l}^{m}$ is unknown which can be determined by applying (8) on $N$ collocation nodes $y^{m},(m=1, \ldots, N)$. The collocation nodes are the nodes on the boundary $S$ and the nodes inside the domain $\Omega$.

With the approximation (8), the domain integral in (4) becomes

$$
\int_{\Omega} u_{k i}^{*}(x, y) g_{i}(y) d \Omega=\sum_{m=1}^{N+A} \alpha_{l}^{m} \int_{\Omega} u_{k i}^{*}(x, y) f^{m}(x) \delta_{i l} d \Omega
$$

The new auxiliary velocity field $\left(\hat{u}_{i}^{l m}(x), \hat{p}^{l m}(x)\right)$ is defined by the following equations

$$
\begin{gathered}
\mu \frac{\partial^{2} \hat{u}_{i}^{l m}(x)}{\partial x_{j} \partial x_{j}}-\frac{\partial \hat{p}^{l m}(x)}{\partial x_{i}}=f^{m}(x) \delta_{i l} \\
\frac{\partial \hat{u}_{i}^{l m}}{\partial x_{i}}=0
\end{gathered}
$$

Applying the Green's identity to the new flow field $\left(\hat{u}_{i}^{l m}(x), \hat{p}^{l m}(x)\right)$ yields

$$
\begin{aligned}
\hat{u}_{i}^{l m}(x)= & \int_{S} t_{k i}^{*}(x, y) \hat{u}_{i}^{l m}(y) d S_{y} \\
& -\int_{S} u_{k i}^{*}(x, y) \hat{t}_{i}^{l m}(y) d S_{y}+\int_{\Omega} u_{k i}^{*}(x, y) f^{m}(y) \delta_{i l} d \Omega
\end{aligned}
$$

where the traction $\hat{t}_{i}^{l m}$ is defined as

$$
\hat{t}_{i}^{l m}(y)=\sigma_{i j}\left(\hat{u}_{i}^{l m}(y), \hat{p}^{l m}(y)\right) n_{j}(y)
$$


Rearranging the terms in (12) we have the transformation from the domain integral to the boundary integral

$$
\begin{aligned}
\int_{\Omega} u_{k i}^{*}(x, y) f^{m}(y) \delta_{i l} d \Omega= & -\int_{S} t_{k i}^{*}(x, y) \hat{u}_{i}^{l m}(y) d S_{y} \\
& +\int_{S} u_{k i}^{*}(x, y) \hat{t}_{i}^{l m}(y) d S_{y}+\hat{u}_{i}^{l m}(x)
\end{aligned}
$$

Substitution of (14) and (9) into (4) leads to integral representation formula in which only boundary integrals are present

$$
\begin{aligned}
& u_{i}(x)-\int_{S} t_{k i}^{*}(x, y) u_{i}(y) d S_{y}+\int_{S} u_{k i}^{*}(x, y) t_{i}(y) d S_{y} \\
& =\sum_{m=1}^{N+A} \alpha_{l}^{m}\left\{-\int_{S} t_{k i}^{*}(x, y) \hat{u}_{i}^{l m}(y) d S_{y}+\int_{S} u_{k i}^{*}(x, y) \hat{t}_{i}^{l m}(y) d S_{y}+\hat{u}_{i}^{l m}(x)\right\}
\end{aligned}
$$

\section{Implementation of the meshless method}

The proposed method will solve at each interior node six integral equations in order to obtain the velocities $u_{1}, u_{2}$, stresses $\sigma_{11}, \sigma_{12}, \sigma_{22}$ and pressure $p$. The integral equation for velocity components is given by (15). Equations for stresses and pressure will be described as follows.

\subsection{Integral equation for stresses}

Stresses are obtained from

$$
\sigma_{k h}=-p \delta_{k h}+\mu\left(\frac{\partial u_{k}}{\partial x_{h}}+\frac{\partial u_{h}}{\partial x_{k}}\right)
$$

The value of derivatives $\partial u_{k} / \partial x_{h}$ are obtained by differentiating (15) in respect to $x_{h}$, where $x_{h}$ is the component of $x$.

$$
\begin{aligned}
& \frac{\partial u_{k}(x)}{\partial x_{h}}=\int_{S} \frac{\partial t_{k i}^{*}(x, y)}{\partial x_{h}} u_{i}(y) d S_{y}-\int_{S} \frac{\partial u_{k i}^{*}(x, y)}{\partial x_{h}} \sigma_{i j}(y) n_{j}(y) d S_{y} \\
& \quad+\sum_{m=1}^{N_{r}+A} \alpha_{l}^{m}\left\{-\int_{S} \frac{\partial t_{k i}^{*}(x, y)}{\partial x_{h}} \hat{u}_{i}^{l m}(y) d S_{y}+\int_{S} \frac{\partial u_{k i}^{*}(x, y)}{\partial x_{h}} \hat{t}_{i}^{l m}(y) d S_{y}+\frac{\partial \hat{u}_{k}^{l m}(x)}{\partial x_{h}}\right\}
\end{aligned}
$$


Substituting (17) into (16) yields equation for $\sigma_{k h}(x)$

$$
\begin{aligned}
\frac{\sigma_{k h}}{\mu}= & -\frac{p}{\mu} \delta_{k h}+\int_{S}\left[\frac{\partial t_{k i}^{*}(x, y)}{\partial x_{h}}+\frac{\partial t_{h i}^{*}(x, y)}{\partial x_{k}}\right] u_{i}(y) d S_{y} \\
& -\int_{S}\left[\frac{\partial u_{k i}^{*}(x, y)}{\partial x_{h}}+\frac{\partial u_{h i}^{*}(x, y)}{\partial x_{k}}\right] \sigma_{i j}(y) n_{j}(y) d S_{y} \\
& +\sum_{m=1}^{N_{r}+A} \alpha_{l}^{m}\left\{-\int_{S}\left[\frac{\partial t_{k i}^{*}(x, y)}{\partial x_{h}}+\frac{\partial t_{h i}^{*}(x, y)}{\partial x_{k}}\right] \hat{u}_{i}^{l m}(y) d S_{y}\right. \\
& \left.+\int_{S}\left[\frac{\partial u_{k i}^{*}(x, y)}{\partial x_{h}}+\frac{\partial u_{h i}^{*}(x, y)}{\partial x_{k}}\right] \hat{t}_{i}^{l m}(y) d S_{y}+\left[\frac{\partial \hat{u}_{k}^{l m}(x)}{\partial x_{h}}+\frac{\partial \hat{u}_{h}^{l m}(x)}{\partial x_{k}}\right]\right\}
\end{aligned}
$$

\subsection{Integral equation for pressure}

The pressure corresponding to the velocity field for the Newtonian fluid can be obtained in the integral form as (for more details, see Ladyzhenskaya [6])

$$
\begin{aligned}
p(x)= & -\int_{S} q^{k}(x, y) t_{j}(y) d S_{y}-2 \mu \int_{S} \frac{\partial q^{k}(x, y)}{\partial x_{j}} u_{k}(y) n_{j}(y) d S_{y} \\
& +\int_{\Omega} q^{k}(x, y) g_{k}(y) d y
\end{aligned}
$$

With the approximation (8), the domain integral in (19) becomes

$$
\int_{\Omega} q^{k}(x, y) g_{k}(y) d \Omega=\sum_{m=1}^{N+A} \alpha_{l}^{m} \int_{\Omega} q^{k}(x, y) f^{m}(x) \delta_{k l} d \Omega
$$

Applying the Green's identity to the new flow field $\left(\hat{u}_{i}^{l m}(x), \hat{p}^{l m}(x)\right)$ produces

$$
\begin{aligned}
\hat{p}^{l m}(x)= & -\int_{S} q^{k}(x, y) \hat{t}_{k}^{l m}(y) d S_{y}-2 \mu \int_{S} \frac{\partial q^{k}(x, y)}{\partial x_{j}} \hat{u}_{k}^{l m}(y) n_{j}(y) d S_{y} \\
& +\int_{\Omega} q^{k}(x, y) f^{m}(y) \delta_{k l} d y
\end{aligned}
$$

Substitution of (21) and (20) into (19) leads to

$$
\begin{aligned}
& p(x)=-\int_{S} q^{k}(x, y) t_{k}(y) d S_{y}-2 \mu \int_{S} \frac{\partial q^{k}(x, y)}{\partial x_{j}} u_{k}(y) n_{j}(y) d S_{y} \\
& +\sum_{m=1}^{N+A} \alpha_{l}^{m}\left(\hat{p}^{l m}(x)+\int_{S} q^{k}(x, y) \hat{t}_{k}^{l m}(y) d S_{y}+2 \mu \int_{S} \frac{\partial q^{k}(x, y)}{\partial x_{j}} \hat{u}_{k}^{l m}(y) n_{j}(y) d S_{y}\right)
\end{aligned}
$$




\section{Interpolation for the unknown fields}

In order to perform the integration over the local boundaries of the circular subdomains, values of the velocities and stresses must be known on the circles. The unknown velocity at any node on the local boundary is approximated by $N$ neighboring nodes by the following formula:

$$
u(x)=\sum_{i=1}^{N} f\left(x, x_{i}\right) \cdot a_{i}
$$

Equation (23) is applied on all $N$ neighboring nodes $x_{j}, j=1, \ldots, N$ and the following system of equations is formed

$$
u\left(x_{j}\right)=\sum_{i=1}^{N} f\left(x_{j}, x_{i}\right) \cdot a_{i}
$$

Equation (24) can be written in the matrix form as

$$
\mathbf{u}_{\mathbf{0}}=\mathbf{F}_{\mathbf{0}} \mathbf{a}
$$

where $\mathbf{u}_{\mathbf{0}}=\left[u\left(x_{1}\right), u\left(x_{2}\right), \ldots, u\left(x_{N}\right)\right]^{T} ; \mathbf{F}_{\mathbf{0}}=\left[f_{j i}\right]=\left[f\left(x_{j}, x_{i}\right)\right], j=1,2$, $\ldots, N ; i=1,2, \ldots, N$. The unknown coefficients $\mathbf{a}$ are determined by $\mathbf{a}=$ $\mathbf{F}_{\mathbf{0}}^{-\mathbf{1}} \mathbf{u}_{\mathbf{0}}$. Hence, the potential at point $x$ can be written as

$$
u(x)=F(x) \mathbf{F}_{\mathbf{0}}^{-\mathbf{1}} \mathbf{u}_{\mathbf{0}}
$$

where $F(x)=\left[f\left(x, x_{1}\right), f\left(x, x_{2}\right), \ldots, f\left(x, x_{N}\right)\right]$.

In the similar way, the stresses are approximated by the following formula

$$
\sigma_{i j}(x)=F(x) \mathbf{F}_{\mathbf{0}}^{-\mathbf{1}} \sigma_{\mathbf{i j}}^{\mathbf{0}}
$$

where $\sigma_{\mathbf{i j}}^{\mathbf{0}}=\left[\sigma_{i j}\left(x_{1}\right), \sigma_{i j}\left(x_{2}\right), \ldots, \sigma_{i j}\left(x_{N}\right)\right]^{T}$.

\section{Solution procedures}

The RBIEM generates one circular sub-domain around each of the nodes located inside the domain or on the boundary (see [7]). The eight nodes on the boundary of the sub-domains are introduced as nodes where the velocities and stresses are evaluated using interpolation by employing the surrounding nodes located at centers of surrounding sub-domains. For each sub-domain, the same set of nodes was used for interpolation of the field variables over the circular local boundaries; this means the same set was used for each of the eight nodes on the circle, and for the DRM approximation. This significantly simplifies the search for the neighbouring nodes and saves CPU time. The set of nodes was found by prescribing the required number of nodes in the interpolation/approximation and then the code defined the set by selecting the required number of the nearest nodes to node $i$. 


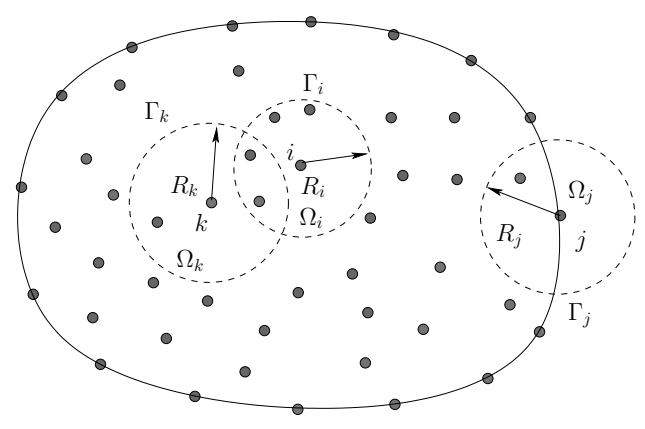

Figure 1: The circular sub-domains distributed in the problem domain.

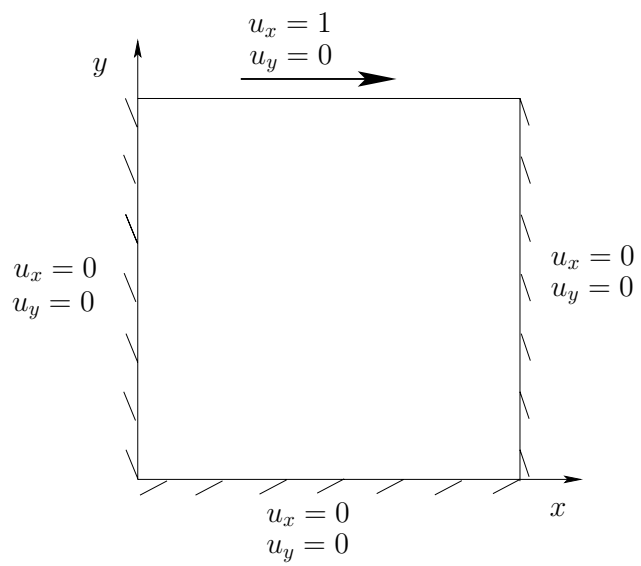

Figure 2: Lid-driven cavity configuration with boundary conditions.

\section{Numerical examples}

The laminar incompressible flow in a square cavity whose upper boundary is moving at a constant velocity (see Figure 2) is considered for benchmarking the numerical approach. The velocity is given as zero on all the walls except at the top, where the horizontal velocity equals to unity. The problem is solved by the proposed method for two different Reynolds number $R e=100, R e=400$. The results obtained by the proposed method are compared with the benchmark values obtained by Ghia et al. [1], using a finite difference multigrid numerical scheme with very fine mesh. For the both cases, the radius of the sub-domain for boundary nodes is set as small as 5.10-3 to reduce the error due to the extrapolation by reducing the distance between the nodes on the external part of the local circular boundary. The radius of the sub-domain for internal nodes is the distance to the nearest node. All simulations are done on a PC Pentium IV 3.0 Ghz, 1.0 Gb of RAM. The resulting sparse matrix produced by the method is solved by SPARSKIT solver. 


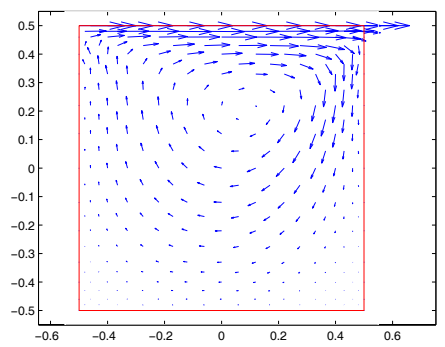

Figure 3: Velocity field at $R e=100$ obtained with 313 nodes in the domain.

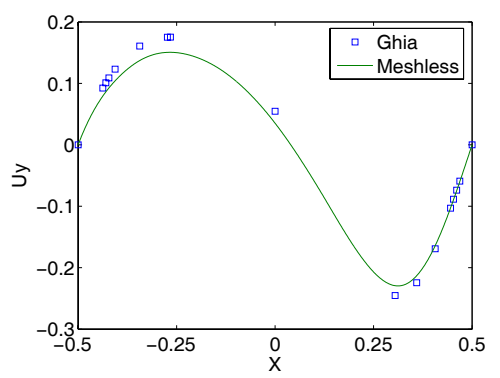

Figure 5: Horizontal fluid velocity distribution at the vertical center line, at $R e=$ 100 with 313 nodes in the domain.

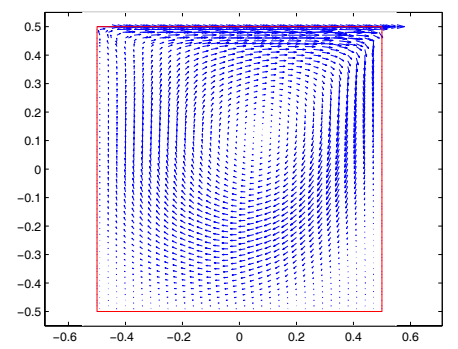

Figure 4: Velocity field at $R e=400$ obtained with 5951 nodes in the domain.

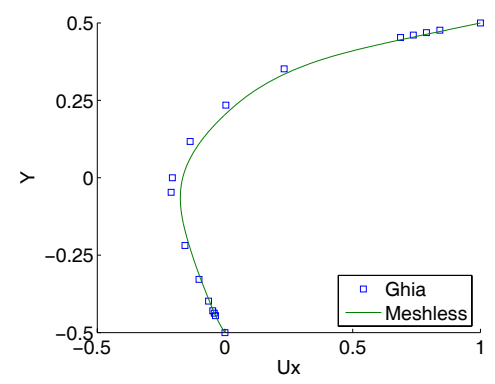

Figure 6: Vertical fluid velocity distribution at the horizontal center line, at $R e=$ 100 with 313 nodes in the domain.

The domain for the case $R e=100$ has 313 nodes. Velocities of the flow for the case $R e=100$ are depicted in figure 3. Figures 5 and 6 show horizontal fluid velocity distribution at the vertical center line and vertical fluid velocity distribution at the horizontal center line, respectively. They are in good agreement with the results obtained by Ghia et al. [1].

As pointed out by Power and Mingo [8], at the flow regions near the corners, the flow has singularities associated with infinite values of the velocity and surface traction. One way to circumvent these problems is to use a high density of boundary elements in the vicinity of the singular points. In the case $R e=400$ there are 5951 nodes in the domain. Figure 4 shows the obtained velocity filed which is in good agreement with previous method. Figures 7 and 8 show a comparison between velocities obtained by proposed approach and those reported by Ghia et al. [1] on the horizontal and vertical center-line fluid. Good agreement of the results has been archived. 


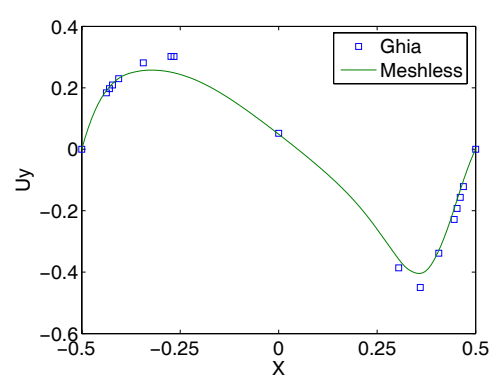

Figure 7: Horizontal fluid velocity distribution at the vertical center line, at $R e=400$ with 5951 nodes in the domain.

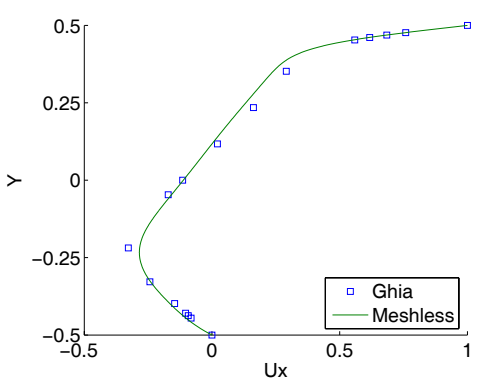

Figure 8: Vertical fluid velocity distribution at the horizontal center line, at $R e=400$ with 5951 nodes in the domain.

\section{Conclusions}

A meshless method based on the integral equations and combined with the subdomain approach is applied to the Navier-Stokes equation. Six equations in 2D are solved at each node, where two equations are for velocities, one equation is for pressure and the remaining equations are used for solving stresses. Radial basis function interpolation is applied in order to obtain the values of the field variable and normal derivatives on the boundary of the circular sub-domains. The inversion of the matrix is calculated only once for every nodal point. Every nodal point is connected with few surrounding nodal points, leading to a banded system. The DRM has been applied to convert the domain integrals into integrals over the boundary of the sub-domains. The numerical results produced by meshless method for two cases $R e=100$ and $R e=400$ are in good agreement with the results obtained by Ghia et al. showing that proposed method can be used for solving the Navier-Stokes equations.

\section{References}

[1] Ghia, U., Ghia, K. \& Shin, C., High-re solutions for incompressible flow using the Navier-Stokes equations and a multigrid method. Journal of Computational Physics, 48, pp. 387-411, 1982.

[2] Zhu, T., Zhang, J. \& N.Atluri, S., A local boundary integral equation (LBIE) method in computational mechanics, and a meshless discretization approach. Computational Mechanics, 21, pp. 223-235, 1988.

[3] Zhu, T., Zhang, J. \& N.Atluri, S., A meshless local boundary integral equation (LBIE) method for solving nonlinear problems. Computational Mechanics, 22, pp. 174-186, 1998. 
[4] Sellountos, E.J. \& Sequeira, A., An advanced meshless LBIE/RBF method for solving two-dimensional incompressible fluid flows. Computational Mechanics, 44, pp. 617-631, 2008.

[5] Bui, T.T. \& Popov, V., The Radial Basis Integral Equation Method for Convection-Diffusion Problems. Ashurst Lodge, Southampton, UK, 2009.

[6] Ladyzhenskaya, O.A., The Mathematical Theory of Viscous Incompressible Flow. Gordon and Breach, New York, 1963.

[7] Popov, V. \& Bui, T.T., A meshless solution to convection-diffusion problems. Submited to Engineering Analysis with Boundary Elements, 2009.

[8] Power, H. \& Mingo, R., The DRM subdomain decomposition approach to solve the two-dimensional Navier-Stokes system of equations. Engineering Analysis with Boundary Elements, 24(1), pp. 107-119, 2000. 